\title{
The end is nigh
}

Friedrich C. Luft

Published online: 22 June 2010

(C) Springer-Verlag 2010

The End Is Nigh is an annual British fanzine that deals with various apocalypses. In this issue, we deal with chromosomal ends for which the end is evidently not yet nigh. The first study linking shortened telomeres to coronary disease lies almost a decade back [1]. Now Maubaret et al. [2] revisit this issue. Telomeres are the regions of repetitive DNA at the end of a chromosome, which protect the end of the chromosome from deterioration. The name is derived from the Greek nouns telos $(\tau \lambda \circ \varsigma)$ and meros $(\mu \varepsilon \dot{\varepsilon} \rho \varsigma)$, namely, the end part. Olovnikov was the first to recognize the problem of how chromosomes could replicate right to the tip, a process that was impossible with replication in a $5^{\prime}$ to $3^{\prime}$ direction. To solve this dilemma and to accommodate Hayflick's idea of limited somatic cell division, Olovnikov suggested that DNA sequences would be lost in every replicative phase until they reached a critical level, at which point, cell division would stop [3, 4].

During cell division, enzymes that duplicate the chromosome and its DNA cannot continue their duplication all the way to the end of the chromosome [5]. If cells divided without telomeres, they would lose the ends of their chromosomes and the necessary information they contain. In 1972, Watson named this phenomenon the "end replication problem." The telomeres are disposable buffers blocking the ends of the chromosomes and are consumed during cell division and replenished by an enzyme, the telomerase reverse transcriptase (telomerase). Telomeres have been likened to the aglets (tips) on the ends of shoelaces that keep them from fraying. Blackburn et al. discovered the unusual nature of telomeres, with their simple repeated DNA sequences composing chromosome

\section{F. C. Luft $(\bowtie)$}

Experimental and Clinical Research Center, Charité Medical Faculty and Max-Delbrück Center for Molecular Medicine, Berlin-Buch, Germany

e-mail: luft@charite.de ends in Tetrahymena. Their work was published in 1978 [6]. Szostak and Blackburn then constructed a linear yeast plasmid by joining fragments from termini of Tetrahymena ribosomal DNA to a yeast vector. They used restriction mapping and hybridization analysis and demonstrated that these fragments were yeast telomeres, which led them to suggest that all yeast chromosomes might also have a common telomere sequence [7]. Greider and Blackburn reported the existence of telomerase in 1985 [8]. The telomere shortening mechanism normally limits cells to a fixed number of divisions, and animal studies suggest that this shortening is responsible for aging on the cellular level and sets a limit on life spans. Telomeres also protect a cell's chromosomes from fusing with each other or rearrangement abnormalities, which can lead to cancer. Cells are normally destroyed when their telomeres are consumed. Most cancers are the result of "immortal" cells that have ways of evading this programmed destruction [5]. Blackburn, Szsotak, and Greider were awarded the Nobel Prize for their work in 2009 (Fig. 1).

Subsequently, telomere sequencing from many organisms including man revealed the presence of repetitive oligomeres, with a high $\mathrm{G}$ content in the strand with its $3^{\prime}$ end at the terminal chromosome. The human telomererepeat sequence is TTAGGG. This sequence is repeated a few thousand base pairs. The $3^{\prime}$ end of the G-rich strand extends 12-16 nucleotides beyond the $5^{\prime}$ end of the complementary C-rich strand. Specific proteins that protect the ends of linear chromosomes from attack by exonucleases bind this region. Specialized regions at the ends of chromosomes are needed, particularly considering that all known DNA polymerases elongate DNA chains at the 3' end, and all require an RNA or DNA primer. As the end of the replication fork approaches the end of a linear chromosome, synthesis of the leading strand continues to the end of the DNA template strand, which completes one 


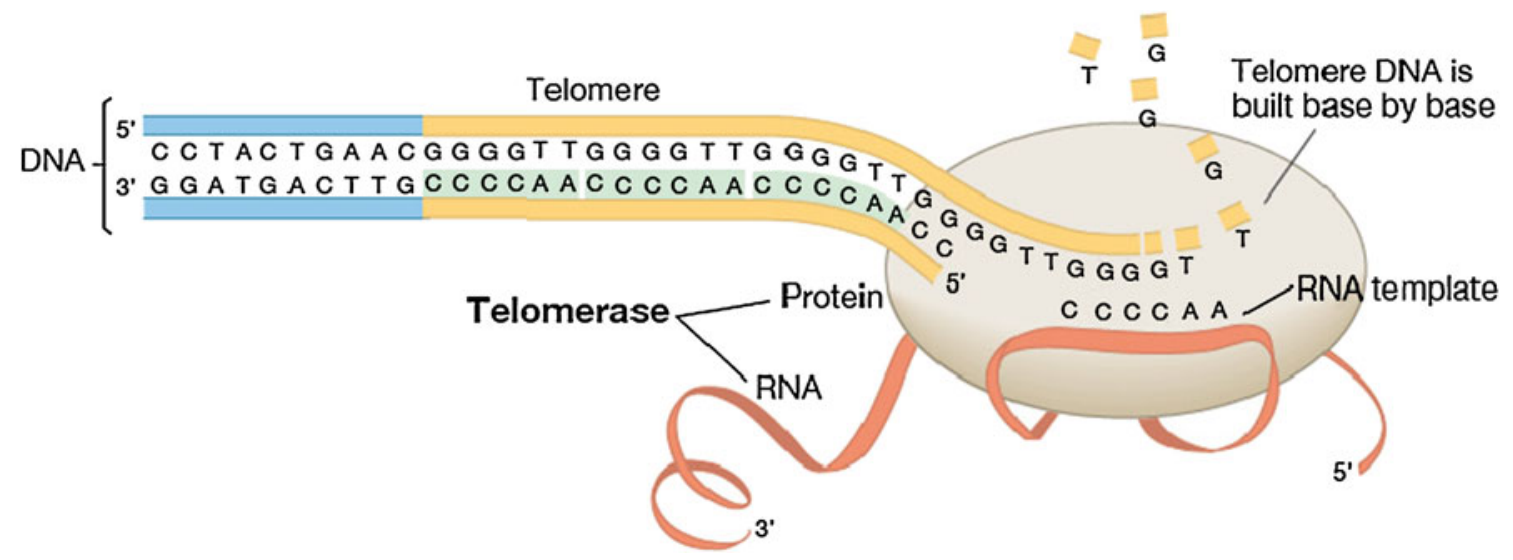

Fig. 1 The DNA sequence varies slightly between species. The one shown here is from Tetrahymena that does not have the same repetitive sequence as man. Telomerase operates at the end of the chromosome. The telomerase enzyme consists of a protein and an

daughter DNA double helix. However, because the lagging strand template is copied in a discontinuous fashion, it cannot be replicated in its entirety. When the final RNA primer is removed, there is no upstream strand onto which DNA polymerase can build to fill the resulting gap. As a result, the leading strand beats the lagging strand by about $8 \mathrm{bp}$ at each division.

Cohen et al. revealed the protein composition of human telomerase in 2007 [9]. The complex consists of two molecules (each) of human telomerase reverse transcriptase (TERT), telomerase noncoding RNA (TR or TERC) and dyskerin (DKC1) [10]. The genes of telomerase subunits, which are TERT, TERC, DKC1, and TEP1, are located on the different chromosomes in human genome. The human TERT gene is translated into a protein of 1,132 amino acids. TERT proteins from many eukaryotes have been sequenced. The TERT protein folds with TERC, which has 451 nucleotides in humans. Reverse transcriptases create singlestranded DNA using single-stranded RNA as a template.

An additional recent novelty is the fact that telomere length is inherited with resetting of the telomere set point. Chiang et al. recently determined that telomere length in offspring is determined by the lengths of their parents' telomeres in the presence of normal telomerase expression [11]. Briefly, they studied models of telomerase haploidinsufficiency in humans and mice to analyze regulation of telomere length and the significance of "set points" in inheritance of telomere length. They first investigated three TERT-mutant families. Some but not all first- and secondgeneration genotypically wild-type offspring of affected TERT-mutated parents had shorter telomeres than did normal age-matched controls. They next analyzed Tert wild-type, Tert gene-deleted, and Tert $+/-$ mice. Their findings suggest that the set point of telomere lengths of offspring is determined by the telomere lengths of their
RNA sequence. The RNA serves as a template for synthesizing telomere DNA (with permission: (C2009 The Nobel Committee for Physiology or Medicine, Karolinska Institutet, SE-171 77 Stockholm, Sweden. Illustration: Annika Röhl, Bengt Gullbing)

parents in the presence of normal expression of telomerase. A Terc gene-deleted mouse has also been generated, which should increase our knowledge even further [12].

The article by Maubaret et al. [2] concerns the finding that patients with myocardial infarction have shorter telomeres than controls. They compared 598 myocardial infarction patients with 653 age-matched UK controls and found that patients with coronary heart disease have shorter telomeres. They included some forms of Mendelian (familial hypercholesterolemia) and polygenic forms of coronary heart disease to make their point. There are critical questions opened immediately by such findings. Are telomeres directly associated with heart disease? Does myocardial infarction, LDL cholesterol, smoking, or whatever causes heart disease shorten telomeres and thereby facilitate the process? Can we manipulate telomere length by stopping smoking, taking statins, or starting a jogging program for instance? What is the influence of glucose levels, type- 2 diabetes, metabolic syndrome, etc. on telomeres? Are persons with a propensity to short telomeres more prone to cardiovascular risk factors? Cardiovascular risk is known to be telomere dependent [5]. Normotensive persons with short telomeres are more likely to develop hypertension. Subclinical activation of the renin-angiotensin system has also been associated with short telomeres. Cigarette smokers have shorter telomeres than nonsmokers. The findings of Maubaret et al. are probably not the last word on the subject [2]. We are left with the interesting question if outcomes for any given complex disease could be influenced by telomerase activity and the actions of TERT. Could TERT become a drug target? Will personalized medicine and human genome sequencing include telomere length in the evaluation? Causal involvement could cause telomere length and telomerase to become targets for preventative and therapeutic strategies. 


\section{References}

1. Samai JN, Boultby R, Butler R, Thompson JR, Goodall AH (2001) Telomere shortening in atherosclerosis. Lancet 358:472-473

2. Maubaret CG, Salpea KD, Jain A, Cooper JA, Hansten A, Sanders J, Montgomery H, Neil A, Nair D, Humphries SE (2010) Telomerers are shorter in myocardial infarction patients compared to health subjects: correlation with environmental risk factors. J Mol Med (current issue) doi:10.1007/s00109-010-0624-3

3. Olovnikov AM (1971) "Принцип маргинотомии в матричном синтезе полинуклеотидов [Principle of marginotomy in template synthesis of polynucleotides]" (in Russian). Dokl Akad Nauk SSSR 201(6):1496-1499

4. Olovnikov AM (1973) A theory of marginotomy. The incomplete copying of template margin in enzymic synthesis of polynucleotides and biological significance of the phenomenon. J Theor Biol 41 (1):181-190. doi:10.1016/0022-5193(73)90198-7, PMID 4754905

5. Oeseburg H, de Boer RA, van Gilst WH, van der Harst P (2010) Telomere biology in healthy aging and disease. Pflugers Arch 459:259-268
6. Blackburn EH, Gall JG (1978) A tandemly repeated sequence at the termini of the extrachromosomal ribosomal RNA genes in Tetrahymena. J Mol Biol 120:33-53

7. Szstak JW, Blackburn EH (1982) Cloning yeast telomeres on linear plasmid vectors. Cell 29:245-255

8. Greider CW, Blackburn EH (1985) Identification of a specific telomere terminal transferase activity in Tetrahymena extracts. Cell 43:405-413

9. Cohen S, Graham M, Lovrecz G, Bache N, Robinson P, Reddel R (2007) Protein composition of catalytically active human telomerase from immortal cells. Science 315:1850-1853

10. Blasco MA (2005) Telomeres and human disease: aging, cancer, and beyond. Nat Rev Genet 6(8):611-622

11. Chiang YJ, Calado RT, Hathcock KS, Lansdorp PM, Young NS, Hodes RJ (2010) Telomere length is inherited with resetting of the telomere set-point. Proc Natl Acad Sci U S A 107(22):10148-53

12. Wong LS, Oeseburg H, de Boer RA, van Gilst WH, van Veldhuisen DJ, van der Harst P (2009) Telomere biology in cardiovascular disease: the TERC-/- mouse as a model for heart failure and ageing. Cardiovasc Res 81:244-252 